\title{
La adaptación de los planes de estudio de Periodismo al EEES. Propuesta metodológica apli- cada a la Universidad de Valladolid
}

\author{
Pilar SÁNCHEZ GARCÍA \\ Universidad de Valladolid (España) \\ pilar.sanchez@hmca.uva.es
}

\begin{abstract}
Resumen
El artículo analiza el cambio de los planes de estudio de Periodismo con la adaptación al Espacio Europeo de Educación Superior. Se presenta una propuesta metodológica con un análisis comparado entre las asignaturas de la Licenciatura y el Grado aplicado a la Universidad de Valladolid en cuatro áreas: eje temático, carácter teórico-práctico, tipo de materia y relación de continuidad. Los resultados evidencian que el nuevo título de Grado renueva su oferta programática en una cuarta parte, incrementa las asignaturas especializadas, prácticas y obligatorias, en detrimento de las teóricas, humanísticas y transversales.
\end{abstract}

Palabras Clave: EEES; Periodismo; Formación; Planes de Estudio; Licenciatura; Grado

Adapting Journalism curricula EEES. Proposed methodology applied to the University of Valladolid

\begin{abstract}
The article analyzes the changing curricula of Journalism with adaptation to the European Higher Education Area. We present a methodological proposal with a comparative analysis between the subjects of Bachelor Degree and the new Degree applied to the University of Valladolid in four areas: thematic, theoretical and practical, material type and relationship continuity. The results show that the new graduate Degree renews a quarter from the Bachelor, increases more specialized subjects, the practical and mandatory in detriment of theoretical, humanistic and cross curricular.
\end{abstract}

Key Words: EEES; Journalism; Education; Curricula; Degree

\section{Referencia normalizada:}

Sánchez García, P. (2013) La adaptación de los planes de estudio de Periodismo al EEES. Propuesta metodológica aplicada a la Universidad de Valladolid. Historia y Comunicación Social. Vol. $18 \mathrm{~N}^{\mathrm{o}}$ Especial Noviembre. Págs. 87-100.

Sumario: 1. Introducción. 2. Metodología. 3. Las necesidades formativas del periodista: más teoría y humanidades o más práctica y especialización. 4. Los cambios programáticos del Espacio Europeo de Educación Superior (EEES). 5. Propuesta metodológica aplicada a la Universidad de Valladolid. 5.1. Instrumentos de medida. 5.2. Cuantificación de resultados. 6. Conclusiones. 7. Bibliografía. 


\section{Introducción}

Los estudios de Periodismo acaban de cumplir 40 años en la Universidad Española (1971-2011) y la reciente convergencia europea de los programas educativos supone un punto de inflexión para analizar el devenir de esta titulación y conocer cuál es la formación que se está ofreciendo en España a los futuros periodistas. La adaptación de los estudios universitarios al Espacio Europeo de Educación Superior (EEES), implantado de forma obligatoria en el curso 2010-2011, tiene ya su primer efecto directo: el cambio de los planes de estudio al pasar de la titulación de Licenciatura al Grado. Aún sin suficiente perspectiva para conocer otras posibles consecuencias como sus resultados pedagógicos y formativo-laborales, sí resulta posible y quizás oportuno analizar uno de los primeros efectos cuantificables en el llamado 'Plan Bolonia', como es el cambio de materias

La titulación de Periodismo se ha adaptado al Grado en las 35 Universidades ${ }^{1}$ que lo imparten en España en la actualidad y ha experimentado cambios que se tratan de analizar y cuantificar en esta investigación con el objetivo de comprobar el nivel de variación o continuidad entre el título de Licenciatura y el de Grado tras la adaptación europea. Se pretende comprobar qué parte educativa se potencia y cuál se considera prescindible, además de analizar en qué materias incide la nueva titulación en la formación de los periodistas españoles. Se parte de la hipótesis de que los nuevos planes del Grado se ajustan a las directrices del llamado Plan Bolonia de manera que, a grandes rasgos, aumentan las materias prácticas y específicas en detrimento de las teóricas, transversales y humanísticas.

Los efectos del EEES comienzan ahora a estudiarse desde diferentes perspectivas en España. Un ejemplo de ello son las últimas investigaciones coordinadas por Javier Sierra Sánchez (2010) sobre los estudios de Comunicación en el EEES, además de otros análisis que se centran en diferentes campos de los nuevos planes de estudio, como ya han hecho Alicia Tapia (2011); Barranquero y Redondo (2009), Rodríguez, Herrero y Sánchez (2010), entre otros.

El presente artículo forma parte de un estudio más amplio en torno a la formación de los periodistas en España ${ }^{2}$ centrado en conocer el devenir de estas enseñanzas en el último siglo, un asunto estudiado por investigadores como Ángel Benito (1967), Enrique de Aguinaga (1984), Marisa Aguirre (1988), María Luisa Humanes (1997), Mercedes Gordon (1991), José Juan Videla (2002) y Elena Real (2004), principalmente. Todos ellos coinciden en la defensa de la formación universitaria del perio-

1 Cifra obtenida a partir del listado de la Agencia Nacional de Evaluación de la Calidad y Acreditación, ANECA (http://srv.aneca.es/ListadoTitulos/busqueda-titulaciones) de la que se excluyen las Facultades que no ofrecen Periodismo como titulación independiente.

2 Es parte de una tesis doctoral sobre la formación de los periodistas españoles que incluye un análisis comparativo entre la Licenciatura y el Grado en las 35 Facultades que imparten Periodismo en la actualidad. 
dista desde un punto de vista integral, sólido y con una perspectiva ética que permita a los informadores desempeñar su trabajo de gran responsabilidad social.

A partir de estos estudios se ahonda en una perspectiva actualizada de la enseñanza periodística en el siglo XXI centrada en los efectos programáticos del EEES en la titulación de Periodismo en España.

\section{Metodología}

La presente investigación recurre a los criterios metodológicos establecidos por R.D Wimmer y J.R. Dominick sobre medios de comunicación (1996). La parte central de la misma es la propuesta metodológica cuantitativa que permite analizar los efectos programáticos del cambio de la Licenciatura al Grado en Periodismo. Pero viene precedida de dos apartados de carácter teórico que muestran el estado de la cuestión: primero, con una aproximación al debate aún inconcluso de la necesidad de formación práctica, humanística, teórica o especializada de los periodistas y, en segundo lugar, con una breve exposición de las principales directrices que establece el EEES en la nueva titulación de Grado. Dicho marco conceptual supone, en realidad, la contextualización de los diferentes rangos o variables que se estudian en el análisis cuantitativo para, a su vez, responder con porcentajes cuantificables a dicho debate teórico, al objeto de aportar conclusiones lo más objetivas posibles sobre cómo es la formación que, en realidad, se ofrece a los periodistas en el siglo XXI en España.

La propuesta metodológica central recurre a la técnica cuantitativa de análisis de contenido, un procedimiento sistemático ideado para examinar una información archivada mediante la creación de categorías que conducen a la obtención de datos objetivos. Se considera la metodología más idónea para analizar los programas de estudio de la Licenciatura y el Grado de Periodismo a través de categorías cuantificables porque ofrece ventajas tanto en la selección de contenidos y variables como en la codificación y análisis de los datos dirigidos a alcanzar conclusiones estadísticas alejadas de impresiones subjetivas (Wimmer y Dominick, 1996).

Esta metodología se aplica al análisis comparativo de los planes de estudio de la Licenciatura y del Grado en Periodismo de la Universidad de Valladolid (por ser donde se realiza esta investigación) tomando como referencia su último programa de Licenciatura (vigente hasta 2015) y el primero del Grado (2010-2011). El modelo propuesto incluye hasta diez variables medibles sobre las materias nuevas que se introducen y las que se suprimen, la incidencia de la formación teórica o práctica, así como el carácter periodístico o de formación general que imprime cada programa y la incorporación de asignaturas nuevas.

Su aplicación a la titulación de Periodismo que se imparte en Valladolid evidencia ciertos cambios en la formación de los periodistas con el llamado Plan Bolonia que acentúa el carácter práctico de sus materias, aumenta las más especializadas en materia comunicacional, en detrimento de los conocimientos generales y humanísticos. 
Los datos indican que la titulación se ha adaptado al carácter más práctico y técnico que marca Espacio Europeo de Educación Superior (EEES).

La propuesta metodológica que se presenta en esta investigación se considera extrapolable al resto de las Facultades españolas que ofrecen la titulación de Periodismo, puesto que en sus cambios de planes de estudio todas ellas toman como base las directrices marcadas por la ANECA (2005) como guía en la adaptación programática al EESS.

\section{Las necesidades formativas del periodista: más teoría y humanidades o más práctica y especialización}

El ámbito académico y profesional del Periodismo arrastra consigo en el último siglo una serie de debates inconclusos que afectan a su planteamiento formativo y a la manera de entender el ejercicio del de la profesión. En la mayoría de los casos, bajo estas discusiones subyacen dos maneras de entender el Periodismo: como oficio eminentemente práctico y de cualidades innatas o como profesión con altas cotas de responsabilidad social que requiere de periodistas altamente cualificados. En este marco se desenvuelve la dicotomía aún irresoluble sobre la formación que deben recibir los profesionales de la información, que se traduce en la carga lectiva teórica o práctica que necesitan, y las enseñanzas humanísticas o especializadas que deben recibir.

Estas posturas responden, a grandes rasgos, a dos modelos internacionales de formación: una corriente teórica y europea, que presta más atención a materias humanísticas y transversales, y otra más práctica y empírica, como es la tradición formativa de EEUU que ha hecho mayor hincapié en la instrucción técnica y prácticas (Díaz Noci, 2005: 85).

Se considera un debate inconcluso o aún abierto porque durante las últimas décadas ha existido la necesidad de revisar las enseñanzas periodísticas. A mediados del XX, con las primeras escuelas y Facultades de Periodismo abiertas en diferentes partes del mundo (especialmente en EEUU, Latinoamérica y Europa), la Unesco reúne a profesionales internacionales en París (1956) que elaboran un informe en el que reclaman la necesidad de ofrecer a los periodistas la más completa formación, "sea cual fuere el medio de información de que se trate" y atendiendo a dos modalidades en busca del equilibrio: una educación práctica, en lo que respecta a las técnicas de la información y una enseñanza teórico-transversal que ofrezca a los periodistas la posibilidad de adquirir un conocimiento lo más amplio posible sobre las cuestiones que hayan de tratar (Unesco, 1957: 13).

Desde entonces, e incluso antes, la duda se centra en la primacía que se debe dar a la transmisión de habilidades técnicas que proporcionen desenvoltura instrumental a los periodistas frente la carga de conocimientos multidisciplinares que ayuden al informador a comprender, contextualizar e interpretar la realidad que le rodea y que retransmite a través de su relato de la actualidad con objetivos interpretativos. En esta última posición se sitúan numerosos investigadores (Ángel Benito, 1967; Aguinaga, 1987; Marisa 
Aguirre, 1988; María Luisa Humanes, 1997; o Mercedes Gordon, 1991, entre otros) cuyas teorías se resumen en una idea:

"El licenciado en Ciencias de la Información no es un humanista, pero su formación debe nutrirse y estar teñida del sentido que dicha formación tiene: preocupación por el estudio del hombre y de la sociedad" (Aguirre, 1988: 273).

Los defensores de una formación integral y transversal consideran de relevancia la enseñanza más técnica pero propugnan un equilibrio entre ambas, es decir, una cultura amplia conjugada con suficientes destrezas profesionales (De Lorenzo, 200: 106). Un equilibrio que se puede considerar como un tercer modelo formativo, el que busca una formación completa y equilibrada con la técnica (López, 2010: 232).

En el ámbito profesional resulta más habitual encontrar argumentos a favor de la formación eminentemente práctica y más especializada en el trabajo periodístico. El investigador y periodista Luis Videla ha revisado estas posturas entre profesionales contemporáneos de reconocido prestigio y cita a Pedro J. Ramírez, Juan Luis Cebrián o Manu Leguineche como ejemplo de una parte de los profesionales que no consideran imprescindible la enseñanza universitaria para trabajar en los medios de comunicación (2002:181-184). En su tesis doctoral, Videla señala que esta postura es un hecho que se mantiene como una constante, con mayor o menor virulencia hasta nuestros días y que se produce por "una visión deformada del trabajo periodístico" (Videla, 2002:117) y de las condiciones en que se debe regular o no esta profesión. Sirva como ejemplo que el veterano periodista Manuel Leguineche critica "la escasa funcionalidad" de los conocimientos humanísticos adquiridos en la Facultad que, en su opinión, deben ser renovados constantemente y sólo resultan útiles en categorías profesionales superiores (1993: 49-50).

Trasladado este viejo debate a la actualidad se puede decir que se reaviva con la revolución que vive el sector con la llegada de las nuevas tecnologías bajo una nueva dicotomía: si los nuevos planes de estudio deben priorizar la formación en medios digitales, con refuerzo de la capacitación instrumental; o si, por el contrario, se debe profundizar en la formación teórica suficiente y amplia como para responder con solvencia informativa en cualquier soporte (Salaverría, 2000: 2-3).

En esta diferencia de posturas ante el nuevo panorama tecnológico se encuentran, de nuevo, dos formas de entender el ejercicio periodístico, desde su función primigenia de transmisor de información a la más compleja labor de intérprete de la realidad. Un cambio que se asienta en la evidencia de que las nuevas tecnologías expanden con mayor facilidad la información desde múltiples canales, de manera que el periodista ha de reforzar su labor de interpretación para distinguirse como profesional. Desde esta perspectiva, el Periodismo de hoy se entiende como un método de interpretación de la realidad social (Gomis, 2008: 56) que requiere de un complejo entramado de conocimientos y saberes. 


\section{Los cambios programáticos del espacio europeo de educación superior (eees)}

La adaptación de los planes de estudio al Espacio Europeo de Educación Superior (EES), conocido como Plan Bolonia por la Declaración firmada en dicha ciudad por 29 ministros de Educación europeos en 1999, establece unas directrices concretas para los nuevos criterios de docencia y evaluación en el cambio de la titulación de Licenciatura a la de Grado. Esas variaciones formativas se basan principalmente en tres aspectos: una mayor movilidad de estudiantes que pueden cursar su carrera en distintos países y la libre circulación de los profesionales de Europa con sus títulos homologados; todo ello a través del tercer pilar de esta reforma, la aplicación de un nuevo método docente en el que las Universidades tendrán mayor libertad para elaborar su oferta educativa.

La nueva titulación se ha implantado de forma obligatoria en España en el curso 2010-2011 y convive con los planes de Licenciatura hasta su extinción prevista en 2015.

A modo de aproximación, y con el objetivo de que puedan comprenderse mejor los resultados del estudio comparativo que se presenta a continuación, se señalan únicamente los principales cambios conceptuales y los nuevos objetivos del plan de convergencia europeo, que van más allá de los conocimientos y se adentran en las habilidades y competencias del alumno.

La primera gran diferencia programática es que cada Universidad cuenta con mayor independencia para programar sus planes de estudio e incluso para modificarlos libremente y para crear nuevas titulaciones. Los cursos se organizan en primer ciclo de Grado (4 años), excepto Arquitectura (5 años) y Medicina (6 años); y un segundo ciclo de Posgrado: Máster (1 o 2 años) y Doctorado (los créditos del máster sustituyen a los cursos de suficiencia investigadora del Doctorado).

En cuanto a las materias se incluyen asignaturas de formación básica (las antiguas troncales; las establece el Ministerio de Educación), obligatorias y optativas; se completan con prácticas externas y con el trabajo fin de grado.

Uno de los cambios sustanciales se encuentra en la distribución de los créditos docentes, puesto que se establece un nuevo cómputo que realmente permite homogeneizar los títulos entre países europeos otorgándoles valores equivalentes para la evaluación del alumno. Se implantan los créditos ECTS (European Credit Transfer System) con la equivalencia de 1 ECTS $=25-30$ horas (dentro y fuera del aula) y 1 año de Grado $=60$ ECTS.

\section{Propuesta metodológica aplicada a la Universidad de Valladolid}

Las nuevas directrices que establece el EEES provocan un cambio inmediato en los programas de estudio. En el caso de la titulación de Periodismo, como ya se ha 
mencionado anteriormente, su adaptación, al igual que en otras titulaciones, implica la supresión de materias, la redistribución lectiva o la incorporación de nuevas asignaturas. Modificaciones que influyen directamente en la formación y en el perfil que se ofrece a los futuros periodistas, de ahí la importancia de conocer la profundidad y características de dicho cambios.

Para ahondar en estas variaciones de la manera más objetiva posible, se presenta una propuesta metodológica aplicada a la Universidad de Valladolid con el objetivo concreto de cuantificar las modificaciones que se dan en el paso de la Licenciatura al Grado de Periodismo. Un modelo de análisis que se considera extrapolable a otros Facultades, lo que permitiría profundizar en los primeros efectos programáticos del EEES en la titulación de Periodismo.

\subsection{Instrumentos de medida}

La propuesta metodológica de tipo cuantitativo recurre a la técnica de análisis de contenido de los planes de estudio de la Licenciatura y del Grado de la Universidad de Valladolid. Se toma como punto de partida un instrumento de medición aplicado a la Universidad Carlos III de Madrid (Barranquero y Redondo, 2009) y se establecen cuatro líneas temáticas que incluyen hasta diez variables aplicadas a las diferentes materias: primero el 'eje temático', si versa sobre Periodismo y Comunicación o es transversal (humanísticas o de cultura general; en segundo lugar se analiza su 'carácter' para ver el contenido práctico o teórico, pero considerando asignaturas eminentemente prácticas las que están encaminadas a 'ensayar' el ejercicio real de la profesión, es decir, las que aportan herramientas instrumentales para su desempeño; en tercer lugar se diferencia el tipo de materia en Troncales, de Formación Básica y Obligatorias; por último, se mide la continuidad de las materias que repiten de la Licenciatura al Grado, las que se suprimen y las que aparecen nuevas.

Entre sus posibles limitaciones se señalan el no incluir las asignaturas optativas y de libre configuración puesto que se considera que, al depender de la elección de cada alumno, podrían desvirtuar el resultado global; así como la diferencia de años de cada titulación ( 5 en Licenciatura y 4 en Grado) que puede considerarse compensado con el cambio de materias anuales a cuatrimestrales.

El modelo de análisis comparativo expuesto se aplica a continuación sobre los planes de estudio de la Licenciatura y el Grado en Periodismo en la Universidad de Valladolid.

Se presenta el análisis mediante tres tablas diferenciadas: la correspondiente al programa de estudios de la Licenciatura (Tabla 1.), del Grado (Tabla 2.) y los resultados porcentuales obtenidos del comparativo entre ambas titulaciones (Tabla 3.) 
Tabla 1. Universidad de Valladolid (Facultad de Filosofía y Letras; Pública). Licenciatura de Periodismo 2003 -309 Créditos (Créditos de optativas: 36. Créditos de Libre configuración: 33).

\begin{tabular}{|c|c|c|c|c|c|c|c|c|c|}
\hline & \multirow[b]{2}{*}{ ASIGNATURA } & \multicolumn{2}{|c|}{ EJE TEMÁTICO } & \multicolumn{2}{|c|}{ CARÁCTER } & \multicolumn{2}{|c|}{ TIPO DE MATERIA } & \multicolumn{2}{|c|}{ CONTINUIDAD } \\
\hline & & $\begin{array}{l}\text { Periodístico / } \\
\text { Comunicac. }\end{array}$ & Transversal & Teórico & Práctico & Troncal & Obligatoria & Sí & No \\
\hline \multirow{7}{*}{ 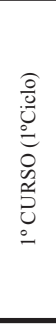 } & $\begin{array}{l}\text { Comunicación e Información Escrita } \\
\text { (12 cr.) }\end{array}$ & $\mathrm{x}$ & & & $\mathrm{x}$ & $\mathrm{x}$ & & $\mathrm{x}$ & \\
\hline & $\begin{array}{l}\text { Comunicación e Información } \\
\text { Audiovisual (12 cr.) }\end{array}$ & $\mathrm{x}$ & & & $\mathrm{x}$ & $\mathrm{X}$ & & $\mathrm{X}$ & \\
\hline & $\begin{array}{l}\text { Teoría de la Comunicación y de la } \\
\text { Información }(12 \mathrm{cr} \text {.) }\end{array}$ & $\mathrm{x}$ & & $\mathrm{x}$ & & $\mathrm{X}$ & & $\mathrm{X}$ & \\
\hline & Lengua Española (9 cr.) & & $\mathrm{x}$ & $\mathrm{X}$ & & $\mathrm{X}$ & & $\mathrm{X}$ & \\
\hline & $\begin{array}{l}\text { Historia Universal Contemporánea } \\
\text { ( } 4.5 \text { cr.) }\end{array}$ & & $\mathrm{x}$ & $\mathrm{x}$ & & $\mathrm{x}$ & & $\mathrm{X}$ & \\
\hline & Historia del Mundo Actual ( $4.5 \mathrm{cr}$.) & & $\mathrm{X}$ & $\mathrm{X}$ & & $\mathrm{X}$ & & $\mathrm{X}$ & \\
\hline & Introducción al Derecho (6 cr.) & & $\mathrm{X}$ & $\mathrm{X}$ & & & $\mathrm{X}$ & $\mathrm{X}$ & \\
\hline \multirow{8}{*}{ 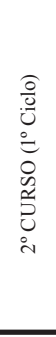 } & Documentación Informativa (6 cr.) & $\mathrm{X}$ & & & $\mathrm{X}$ & $\mathrm{X}$ & & $\mathrm{X}$ & \\
\hline & Teoría de la Publicidad (6 cr.) & $\mathrm{x}$ & & $\mathrm{x}$ & & $\mathrm{x}$ & & & $\mathrm{X}$ \\
\hline & $\begin{array}{l}\text { Fundamentos de las Relaciones } \\
\text { Públicas ( } 6 \text { cr.) }\end{array}$ & $\mathrm{x}$ & & $\mathrm{x}$ & & $\mathrm{x}$ & & & $\mathrm{X}$ \\
\hline & Historia de España Actual (9 cr.) & & $\mathrm{X}$ & $\mathrm{X}$ & & & $\mathrm{X}$ & $\mathrm{X}$ & \\
\hline & $\begin{array}{l}\text { Tecnología de los Medios } \\
\text { Audiovisuales }(9 \mathrm{cr} \text { ) }\end{array}$ & $\mathrm{x}$ & & & $\mathrm{X}$ & & $\mathrm{x}$ & $\mathrm{X}$ & \\
\hline & Redacción Periodística (9 cr.) & $\mathrm{X}$ & & & $\mathrm{X}$ & & $\mathrm{X}$ & $\mathrm{X}$ & \\
\hline & $\begin{array}{l}\text { Géneros Informativos y Producción en } \\
\text { Radio y Televisión }(9 \text { cr.) }\end{array}$ & $\mathrm{x}$ & & & $\mathrm{x}$ & & $\mathrm{x}$ & $\mathrm{X}$ & \\
\hline & Introducción a la Economía (6 cr.) & & $\mathrm{X}$ & $\mathrm{X}$ & & & $\mathrm{X}$ & $\mathrm{X}$ & \\
\hline \multirow{5}{*}{ 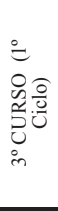 } & $\begin{array}{l}\text { Producción Periodística en Nuevas } \\
\text { Tecnologías ( } 6 \text { cr.) }\end{array}$ & $\mathrm{x}$ & & & $\mathrm{X}$ & & $\mathrm{x}$ & $\mathrm{X}$ & \\
\hline & Diseño Periodístico ( 6 cr.) & $\mathrm{X}$ & & & $\mathrm{X}$ & & $\mathrm{x}$ & $\mathrm{X}$ & \\
\hline & Periodismo Científico (6 cr.) & $\mathrm{X}$ & & & $\mathrm{X}$ & & $\mathrm{X}$ & & $\mathrm{X}$ \\
\hline & $\begin{array}{l}\text { Movimientos Literarios y su } \\
\text { Proyección Periodística (6 cr.) }\end{array}$ & $\mathrm{x}$ & & $\mathrm{X}$ & & & $\mathrm{x}$ & & $\mathrm{X}$ \\
\hline & Introducción a la Geografía (6 cr.) & & $\mathrm{X}$ & $\mathrm{X}$ & & & $\mathrm{x}$ & & $\mathrm{X}$ \\
\hline \multirow{7}{*}{ 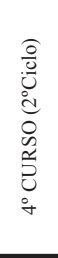 } & Derecho de la Información (9 cr.) & & $\mathrm{X}$ & $\mathrm{X}$ & & $\mathrm{X}$ & & $\mathrm{X}$ & \\
\hline & Periodismo Especializado (12 cr.) & $\mathrm{x}$ & & & $\mathrm{X}$ & $\mathrm{X}$ & & $\mathrm{X}$ & \\
\hline & Empresa Informativa (9 cr.) & $\mathrm{X}$ & & $\mathrm{X}$ & & $\mathrm{X}$ & & $\mathrm{X}$ & \\
\hline & Tecnología de la Información (12 cr.) & $\mathrm{x}$ & & & $\mathrm{X}$ & $\mathrm{X}$ & & $\mathrm{X}$ & \\
\hline & Teoría e Historia del Periodismo (9cr.) & $\mathrm{X}$ & & $\mathrm{X}$ & & $\mathrm{X}$ & & $\mathrm{X}$ & \\
\hline & $\begin{array}{l}\text { Producción Escrita y Audiovisual } \\
(6 \text { cr. })\end{array}$ & $\mathrm{x}$ & & & $\mathrm{X}$ & $\mathrm{x}$ & & $\mathrm{X}$ & \\
\hline & Sociología de la Opinión Pública (6 cr.) & $\mathrm{X}$ & & $\mathrm{X}$ & & & $\mathrm{x}$ & $\mathrm{X}$ & \\
\hline \multirow{4}{*}{ 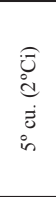 } & $\begin{array}{l}\text { Historia de la Comunicación Social } \\
\text { Contemporánea }(6 \mathrm{cr} \text { ) }\end{array}$ & $\mathrm{X}$ & & $\mathrm{x}$ & & & $\mathrm{x}$ & $\mathrm{X}$ & \\
\hline & $\begin{array}{l}\text { Las Relaciones Internacionales en el } \\
\text { Mundo Actual ( } 6 \text { cr.) }\end{array}$ & & $X$ & $X$ & & & $X$ & $\mathrm{X}$ & \\
\hline & Deontología de la Comunicación (6 cr.) & & $\mathrm{X}$ & $\mathrm{x}$ & & & $\mathrm{x}$ & $\mathrm{X}$ & \\
\hline & $\begin{array}{l}\text { Modelos de Uso de la Lengua Española } \\
\text { ( } 9 \text { cr.) }\end{array}$ & & $\mathrm{X}$ & $\mathrm{x}$ & & & $\mathrm{x}$ & $\mathrm{X}$ & \\
\hline
\end{tabular}

Plan de Estudios de Licenciatura en Periodismo de la Universidad de Valladolid. Elaboración propia 
Tabla 2. Universidad de Valladolid (Facultad de Filosofía y Letras; Pública).Grado de Periodismo 2010 -240 Créditos (Créditos de optativas: 30).

\begin{tabular}{|c|c|c|c|c|c|c|c|c|c|}
\hline & \multirow[b]{2}{*}{ ASIGNATURA } & \multicolumn{2}{|c|}{ EJE TEMÁTICO } & \multicolumn{2}{|c|}{ CARÁCTER } & \multicolumn{2}{|c|}{ TIPO DE MATERIA } & \multicolumn{2}{|c|}{ CONTINUIDAD } \\
\hline & & $\begin{array}{c}\text { Periodístico / } \\
\text { Comunicacional }\end{array}$ & Transversal & Teórico & Práctico & $\begin{array}{l}\text { Formación } \\
\text { Básica }\end{array}$ & Obligatoria & Sí & Nueva \\
\hline \multirow{10}{*}{$\begin{array}{l}0 \\
\hat{W} \frac{0}{0} \\
\tilde{S}_{0} \\
0 \\
0\end{array}$} & Historia del Mundo Actual (6 cr.) & & $\mathrm{X}$ & $\mathrm{X}$ & & $\mathrm{X}$ & & $\mathrm{x}$ & \\
\hline & $\begin{array}{l}\text { Lengua Española Aplicada al Periodismo } \\
(6 \text { cr.) }\end{array}$ & & $\mathrm{x}$ & $\mathrm{x}$ & & $\mathrm{x}$ & & $\mathrm{x}$ & \\
\hline & Teorías de la Comunicación ( 6 cr.) & $\mathrm{X}$ & & $\mathrm{X}$ & & $\mathrm{X}$ & & $\mathrm{X}$ & \\
\hline & Redacción Periodística I (6 cr.) & $\mathrm{X}$ & & & $\mathrm{X}$ & & $\mathrm{X}$ & $\mathrm{X}$ & \\
\hline & $\begin{array}{l}\text { Estructura de los Medios de Comunicación } \\
\text { (6 cr.) }\end{array}$ & $\mathrm{x}$ & & $\mathrm{X}$ & & $\mathrm{x}$ & & $\mathrm{X}$ & \\
\hline & Economía aplicada al Periodismo $(6 \mathrm{cr}$ ) & & $\mathrm{X}$ & $\mathrm{X}$ & & $\mathrm{X}$ & & $\mathrm{X}$ & \\
\hline & $\begin{array}{l}\text { Nuevas Tecnologías aplicadas al Periodismo } \\
\text { (6 cr.) }\end{array}$ & $\mathrm{x}$ & & & $\mathrm{X}$ & & $\mathrm{X}$ & $\mathrm{X}$ & \\
\hline & $\begin{array}{l}\text { Teoría de la Información y de los Efectos } \\
\text { (6 cr.) }\end{array}$ & $\mathrm{x}$ & & $\mathrm{x}$ & & & $\mathrm{x}$ & $\mathrm{X}$ & \\
\hline & Redacción periodística (II) $(6 \mathrm{cr}$.) & & $\mathrm{x}$ & & $\mathrm{x}$ & & $\mathrm{X}$ & $\mathrm{x}$ & \\
\hline & Inglés para periodistas (I) $(6 \mathrm{cr}$.) & & $\mathrm{X}$ & $\mathrm{X}$ & & $\mathrm{X}$ & & & $\mathrm{X}$ \\
\hline \multirow{10}{*}{ 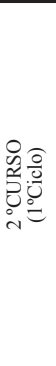 } & Derecho de la Información (6 cr.) & & $\mathrm{X}$ & $\mathrm{X}$ & & $\mathrm{X}$ & & $\mathrm{X}$ & \\
\hline & Ideas Políticas Contemporáneas (6 cr.) & & $\mathrm{X}$ & $\mathrm{X}$ & & $\mathrm{X}$ & & $\mathrm{X}$ & \\
\hline & Radio Informativa (6 cr.) & $\mathrm{X}$ & & & $\mathrm{X}$ & & $\mathrm{X}$ & $\mathrm{X}$ & \\
\hline & Lenguaje Audiovisual (6 cr.) & $\mathrm{X}$ & & & $\mathrm{X}$ & & $\mathrm{X}$ & $\mathrm{x}$ & \\
\hline & Diseño Periodístico (6 cr.) & $\mathrm{X}$ & & & $\mathrm{X}$ & & $\mathrm{X}$ & $\mathrm{X}$ & \\
\hline & $\begin{array}{l}\text { Relaciones Internacionales del Mundo } \\
\text { Contemporáneo (6 cr.) }\end{array}$ & & $\mathrm{x}$ & $\mathrm{x}$ & & $\mathrm{x}$ & & $\mathrm{x}$ & \\
\hline & Documentación Informativa (6 cr.) & $\mathrm{X}$ & & & $\mathrm{X}$ & & $\mathrm{X}$ & $\mathrm{X}$ & \\
\hline & Televisión Informativa (6 cr.) & $\mathrm{X}$ & & & $\mathrm{X}$ & & $\mathrm{X}$ & $\mathrm{X}$ & \\
\hline & Géneros Periodísticos Interpretativos (6 cr.) & $\mathrm{X}$ & & & $\mathrm{X}$ & & $\mathrm{X}$ & $\mathrm{X}$ & \\
\hline & Inglés para periodistas (II) $(6 \mathrm{cr}$.) & & $\mathrm{X}$ & $\mathrm{X}$ & & $\mathrm{X}$ & & & $\mathrm{X}$ \\
\hline \multirow{8}{*}{ 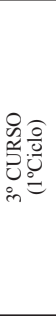 } & Historia del Periodismo Universal (6 cr.) & $\mathrm{X}$ & & $\mathrm{X}$ & & & $\mathrm{X}$ & $\mathrm{X}$ & \\
\hline & Comunicación Organizacional (6 cr.) & $\mathrm{X}$ & & & $\mathrm{x}$ & & $\mathrm{X}$ & & $\mathrm{X}$ \\
\hline & Periodismo de Opinión (6 cr.) & $\mathrm{X}$ & & & $\mathrm{X}$ & & $\mathrm{X}$ & $\mathrm{X}$ & \\
\hline & Ciberperiodismo (6 cr.) & $\mathrm{X}$ & & & $\mathrm{X}$ & & $\mathrm{X}$ & & $\mathrm{X}$ \\
\hline & Historia del Periodismo Español (6 cr.) & $\mathrm{X}$ & & $\mathrm{X}$ & & & $\mathrm{X}$ & $\mathrm{X}$ & \\
\hline & $\begin{array}{l}\text { Gestión y Producción de Proyectos } \\
\text { Periodísticos (6 cr.) }\end{array}$ & $\mathrm{x}$ & & & $\mathrm{x}$ & & $\mathrm{X}$ & $\mathrm{x}$ & \\
\hline & Periodismo Especializado (6 cr.) & $\mathrm{X}$ & & & $\mathrm{X}$ & & $\mathrm{X}$ & $\mathrm{X}$ & \\
\hline & $\begin{array}{l}\text { Metodologías de Investigación en } \\
\text { Comunicación (6 cr.) }\end{array}$ & $\mathrm{x}$ & & $\mathrm{x}$ & & & $\mathrm{X}$ & & $\mathrm{x}$ \\
\hline \multirow{7}{*}{ 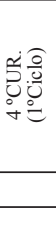 } & Comunicación Política (6 cr.) & $\mathrm{X}$ & & & $\mathrm{X}$ & & $\mathrm{X}$ & $\mathrm{X}$ & \\
\hline & Ética y Deontología Periodística (6 cr.) & & $\mathrm{X}$ & $\mathrm{X}$ & & & $\mathrm{X}$ & $\mathrm{x}$ & \\
\hline & Fotoperiodismo (6 cr.) & $\mathrm{X}$ & & & $\mathrm{X}$ & & $\mathrm{X}$ & & $\mathrm{X}$ \\
\hline & Periodismo de Investigación (6 cr.) & $\mathrm{X}$ & & & $\mathrm{X}$ & & $\mathrm{X}$ & & $\mathrm{X}$ \\
\hline & Información y Derechos Humanos (6 cr.) & $\mathrm{X}$ & & & $\mathrm{X}$ & & $\mathrm{X}$ & & $\mathrm{X}$ \\
\hline & Trabajo Fin de Grado (6 cr.) & $\mathrm{X}$ & & & $\mathrm{X}$ & & $\mathrm{X}$ & & $\mathrm{X}$ \\
\hline & Prácticas Externas (12 cr.) & $\mathrm{X}$ & & & $\mathrm{X}$ & & $\mathrm{X}$ & & $\mathrm{X}$ \\
\hline
\end{tabular}

Plan de Estudios de Grado en Periodismo de la Universidad de Valladolid. Elaboración propia 
Tabla 3. Ficha de análisis: Comparativo Licenciatura y Grado. Universidad de Valladolid

\begin{tabular}{|c|c|c|c|c|c|c|c|c|c|c|}
\hline \multirow[b]{2}{*}{ PLAN DE ESTUDIOS } & \multicolumn{2}{|c|}{ EJE TEMÁTICO } & \multicolumn{2}{|c|}{ CARÁCTER } & \multicolumn{3}{|c|}{ TIPO DE MATERIA } & \multicolumn{3}{|c|}{ CONTINUIDAD } \\
\hline & $\begin{array}{c}\text { Periodístico/ } \\
\text { Comunicacional }\end{array}$ & Transversal & Teórico & Práctico & Troncal & $\begin{array}{c}\begin{array}{c}\text { Formación } \\
\text { Básica }\end{array} \\
\end{array}$ & Obligatoria & $\mathrm{Si}$ & No & Nueva \\
\hline $\begin{array}{l}\text { LICENCIATURA DE } \\
\text { PERIODISMO } \\
\text { ASIGNATURAS } \\
\text { (5 AÑOS)--- } 31\end{array}$ & $\begin{array}{c}20 \\
64,5 \%\end{array}$ & $\begin{array}{c}11 \\
35,5 \%\end{array}$ & $\begin{array}{c}19 \\
61,2 \%\end{array}$ & $\begin{array}{c}12 \\
38,7 \%\end{array}$ & $\begin{array}{c}15 \\
48,3 \%\end{array}$ & & $\begin{array}{c}16 \\
51,6 \%\end{array}$ & $\begin{array}{c}26 \\
83,8 \%\end{array}$ & $\begin{array}{c}5 \\
16,1 \%\end{array}$ & \\
\hline $1^{\circ}, 2^{\circ}, 3^{\circ}$ curso $\rightarrow 20$ & $13 / 41,9 \%$ & $7 / 22,5 \%$ & $11 / 35,5 \%$ & $9 / 29 \%$ & $9 / 29 \%$ & - & $11 / 35,5 \%$ & $15 / 48,3 \%$ & $5 / 16,1 \%$ & - \\
\hline $4^{\circ}$ y $5^{\circ}$ curso $\rightarrow 11$ & $7 / 22,5 \%$ & $4 / 12,9 \%$ & $8 / 25,8 \%$ & $3 / 9,6 \%$ & $6 / 19,3 \%$ & - & $5 / 16,1 \%$ & $11 / 35,5 \%$ & 0 & - \\
\hline $\begin{array}{c}\text { GRADO DE PERIODISMO } \\
\text { ASIGNATURAS--- } 35 \\
\text { (4 AÑOS) }\end{array}$ & $\begin{array}{c}25 \\
71,4 \%\end{array}$ & $\begin{array}{c}10 \\
28,5 \%\end{array}$ & $\begin{array}{c}15 \\
42,8 \%\end{array}$ & $\begin{array}{c}20 \\
57,1 \%\end{array}$ & & $\begin{array}{c}10 \\
28,5 \%\end{array}$ & $\begin{array}{c}23 \\
65,7 \%\end{array}$ & $\begin{array}{c}25 \\
71,4 \%\end{array}$ & & $\begin{array}{c}10 \\
28,5 \%\end{array}$ \\
\hline $1^{\circ}$ y $2^{\circ}$ curso $\rightarrow 20$ & $11 / 31,4 \%$ & $9 / 25,7 \%$ & $11 / 31,4 \%$ & $9 / 25,7 \%$ & - & $10 / 28,5 \%$ & $10 / 28,5 \%$ & $18 / 51,4 \%$ & - & $2 / 5,7 \%$ \\
\hline $3^{\circ}$ y $4^{\circ}$ curso $\rightarrow 15$ & $14 / 40 \%$ & $1 / 2,8 \%$ & $4 / 11,4 \%$ & $11 / 31,4 \%$ & - & 0 & $15 / 42,8 \%$ & $7 / 20 \%$ & - & $8 / 22,8 \%$ \\
\hline
\end{tabular}

Resultado del análisis comparativo entre la Licenciatura y el Grado en Periodismo de la Universidad de Valladolid. Elaboración propia

\subsection{Cuantificación de resultados}

El análisis comparado de los planes de estudio de Licenciatura y Grado en Periodismo en la Universidad de Valladolid (UVA) permite cuantificar sus resultados a partir de las variables analizadas y de las que aquí se presentan de manera sucinta por límites de espacio (pueden verse al completo en la Tabla 3):

En cuanto al 'Eje temático', en ambos planes de estudio predominan las materias especializadas en 'Periodística-Comunicacional' incluso aumentan en el Grado hasta un $71,4 \%$ frente a las consideradas 'Transversales' que se reducen siete puntos, hasta un $28,5 \%$.

Las asignaturas de carácter práctico (encaminadas a la ejercitación profesional) incrementan su presencia hasta representar más de la mitad de la oferta docente, con un $57,1 \%$, frente al 38,7\% de la Licenciatura. Así, la presencia de materias teóricas pasa del $61,2 \%$ al $42,8 \%$ en los nuevos estudios universitarios.

En el Grado aumentan las materias obligatorias, un 65,7\%, que dependen de la libertad de elección de cada Universidad. Y los datos evidencian que se produce una disminución de 18 puntos porcentuales en las propuestas por el Gobierno con el 48,3\% de 'Troncales' de la Licenciatura al 28,5\% de 'Formación Básica' del Grado.

Respecto a la continuidad, el 83,8\% de las asignaturas del antiguo plan se mantienen en la nueva titulación y las que desaparecen son de carácter teórico y obligatorio.

El análisis de estos mismos resultados por cursos, evidencia que las materias con temática periodística y comunicacional están presentes toda la carrera. En la primera mitad de las dos titulaciones (los tres primeros de Licenciatura y los dos primeros del Grado) se concentra mayor número de asignaturas y entre ellas abundan las consideradas transversales, teóricas y obligatorias. En la segunda mitad de las titulaciones se 
concentra la formación más técnica y es donde se incorporan las nuevas materias que ofrecen un contenido más práctico, periodístico y relacionado con las nuevas tecnologías.

\section{Conclusiones}

La investigación realizada sobre la adaptación de los estudios de Periodismo al Espacio Europeo de Educación Superior (EEES) permite establecer unas conclusiones concretas a partir del análisis comparativo realizado entre la Licenciatura y el Grado en Periodismo en la Universidad de Valladolid. Dicho comparativo se presenta como una propuesta metodológica aplicable a otras Facultades que imparten la titulación de Periodismo. Las conclusiones que se presentan a continuación pueden considerarse como un primer diagnóstico de los efectos programáticos del EEES en la formación de periodistas.

En primer lugar se detecta que el primer efecto directo en la adaptación de los planes de estudio de Periodismo al EEES se produce una modificación de las materias teóricas o prácticas y especializadas o transversales.

En segundo término los datos evidencian que la nueva titulación de Grado ofrece un conocimiento más especializado con el aumento de las materias centradas en el área de Periodismo y de la Comunicación, propias de la titulación, en detrimento de la formación considerada humanística con la supresión de materias de cultura general o transversal.

En tercer término se comprueba que las asignaturas encaminadas a la instrucción más técnica de la profesión copan más de la mitad de nueva oferta educativa, con la consiguiente reducción de las estrictamente teóricas que estaban más presentes en los antiguos planes de estudio.

En cuarto lugar, se confirma la mayor autonomía de las Universidades en la elaboración del plan de estudios con mayor presencia de las materias Obligatorias que proponen bajo su propio criterio, disminuyendo las que marca el Ministerio de Educación (Troncales y de Formación Básica).

En quinto término se constata que el cambio de la Licenciatura al Grado no supone una alteración radical del programa puesto que se mantienen las dos terceras partes de las materias en el nuevo programa.

En sexto término, en el análisis por cursos se detecta que adquieren mayor presencia las materias relacionadas directamente con la titulación y las que son de carácter práctico en todos los cursos analizados, de manera que en la segunda mitad del Grado se reducen las de carácter teórico y transversal. La mayor continuidad se da en los primeros años y la renovación en los dos últimos. 
En séptimo lugar, tal y como se esperaba, los resultados evidencian que la nueva titulación de Grado en Periodismo se adapta a las líneas generales del Plan Bolonia con un carácter más práctico y especializado en su área de conocimiento.

\section{Referencias bibliográficas}

AGUINAGA, E. (1980). Periodismo Profesión. Madrid: Fragua.

(1984): Epistemología del ejercicio periodístico. Los estudios de periodismo y su proyección profesional. Tesis Doctoral. Universidad Complutense.

AGUIRRE, M. (1988). El deber de formación en el informador. Pamplona: Eunsa.

BENITO, Á. (1967). "Evolución de los estudios de Periodismo en el mundo". En: Ciencia y enseñanza del Periodismo, Cuadernos de Trabajo, $\mathrm{n}^{\mathrm{o}}$ 11, Pamplona: Universidad de Navarra.

BENITO, Á; FATTORELlO, F. \& GABEL, E. (1967): “Ciencia y Enseñanza del Periodismo". En Cuadernos de Trabajo, no 11, Pamplona: Universidad de Navarra.

DE LORENZO, I. (2007). "El periodismo y la redacción periodística en los manuales de periodismo de 1936 a 197'. En VVAA, La Periodística como disciplina Universitaria: balance y perspectivas, Navarra: Sociedad Española de Periodística.

DÍAZ, J. (2005). "Periodismo en Internet: investigar los nuevos medios", en VVAA, en Investigar sobre Periodismo, Reunión Científica, Santiago de Compostela: Sociedad Española de Periodística.

GOMIS, L. (2008). Teoría de los géneros periodísticos. Barcelona: UOC

HUMANES, M. L. (1998): “La profesión periodística en España”. En: Zer: Revista de estudios de comunicación, $\mathrm{n}^{\circ}$. 4, Madrid: Universidad del País Vasco. (2011). "La enseñanza del Periodismo en España”. En: SIERRA: F. y MORENO F.J: (Ed.), Fundamentos del Teoría del Periodismo. Sevilla: Universidad de Sevilla, pp: $39-47$

LEGUINECHE, M. (1993): El Periodista. Madrid: Acento.

LÓPEZ, X. (2010). "La formación de los periodistas en el siglo XXI en Brasil, España, Portugal y Puerto Rico". En: Revista Latina de Comunicación Social, no65, Tenerife: Universidad de La Laguna, p. 231 a 243.

RODRÍGUEZ, M.I.; HERRERO, F.J.; SÁNCHEZ, F. (2010). "Los medios de comunicación en las redes sociales ante el reto de Bolonia". En SIERRA, J.; CABEZUELO, F. (Coord.), Competencias y perfiles profesionales en los estudios de Ciencias de la Comunicación, Madrid: Fragua, p. 194-205.

SIERRA, J.; CABEZUELO, F. (coords.) (2010). Competencias y perfiles profesionales en los estudios de Ciencias de la Comunicación. Madrid: Fragua.

SIERRA, J. (Coord) (2010). Los estudios de Ciencias de la Comunicación en el EEES. Madrid: Fragua.

SIERRA, J. Y MORENO F.J. (Eds.), (2011). Fundamentos del Teoría del Periodismo. Sevilla: Universidad de Sevilla. 
TAPIA, A. (2011). "Las primeras enseñanzas de documentación en periodismo". En: Documentación de las Ciencias de la Información, $\mathrm{n}^{\circ}$ 24, Madrid: Universidad Complutense, p. 231-253.

WIMMER R.D.; DOMINICK J.R. (1996). La investigación cientifica de los medios de comunicación: una introducción a sus métodos. Barcelona: Bosch.

Consultas en la web

ANECA. Titulaciones con Grado en Periodismo. En http://srv.aneca.es/ListadoTitulos/busqueda-titulaciones [10/01/2012].

(2005). Libro Blanco. Título de Grado en Comunicación. En http://www.aneca.es/ media/150336/libroblancocomunicaciondef.pdf. [03-02-2010].

BARRANQUERO, A. \& REDONDO, F. (2009). "Análisis Comparado de los planes de Estudio de Periodismo antes y después de Bolonia", en Actas del I Congreso Internacional Latina de Comunicación Social. Tenerife. En http://www.revistalatinacs. org/09/Sociedad/actas/14barranquero.pdf. [03-06-2010].

GRAÑA, M. (1927). 'Escuelas de Periodismo' Conferencia pronunciada en la Sociedad de Estudios Vasca. En: http://www.euskomedia.org/PDFAnlt/congresos/04/04240252.pdf [02-02-2012].

GORDÓN, M. (1991) La enseñanza del Periodismo en el mundo occidental. Estudio Comparado de tres escuelas. Tesis doctoral. En http://eprints.ucm.es/1763/ [03-11-2009].

REAL, E. (2004). Formación y ejercicio profesional del periodista en la España del siglo XXI dentro del marco de la Unión Europea. Tesis Doctoral, Universidad Complutense. En http://eprints.ucm.es/tesis/inf/ucm-t27334.PDF [09-05-2011].

SALAVERRÍA, R. (2000). "Criterios para la formación de periodistas en la era digital", en I Congreso Nacional de Periodismo Digital. Huesca, (14-15 de enero de 2000). En http://www.unav.es/fcom/mmlab/mmlab/investig/crite.htm. [17-022013].

UNESCO (1958). La formación de periodistas. Estudio mundial sobre la preparación del personal de la información. París: Unesco. En: http://unesdoc.unesco. org/images/0013/001353/135346so.pdf. [02-04-2012].

(2007). Plan modelo de estudios de periodismo. Francia, UNESCO. En http:// www.unesco.org/new/es/communication-and-information/resources/publications-and-communication-materials/publications/full-list/model-curricula-for-journalism-education/. [05-03-2011].

UNESCO (2013). Enseñanza del Periodismo y Formación de Periodistas. Disponible en: http://www.unesco.org/new/es/communication-and-information/media-development/journalism-education-and-training/ [05-03-2013].

UNIVERSIDAD DE VALLADOLID: "Plan de estudios de la Licenciatura Periodismo". En: http:/www.uva.es/opencms/consultas/planesestudios/asignaturas?codigo plan=313\&ano_academico=1213. [02-02-2010].

"Plan de estudios de Graduado en Periodismo", BOE núm.2 de 3 de enero de 2011. En: http://www.boe.es/boe/dias/2011/01/03/pdfs/BOE-A-2011-142.pdf. [10-06-2012]. 
VIDELA, J.J. (2002). La formación de los periodistas en España: perspectiva histórica y propuestas de futuro. Tesis Doctoral. Madrid. Universidad Complutense. En: http://pendientedemigracion.ucm.es/BUCM/tesis/inf/ucm-t25979.pdf [20-012013].

\section{La autora}

Pilar Sánchez García es Licenciada en Ciencias de la Información por la Universidad Pontificia de Salamanca y profesora de la Universidad de Valladolid. Miembro del equipo de investigación "El 'infoentretenimiento' político en televisión e Internet. Formatos, audiencias y consecuencias en la comunicación política española (INFOPOLNET)" y del "Grupo de Innovación Docente en Comunicación para el desarrollo digital y la inclusión de género (GID-COMDIG)". Sus investigaciones giran en torno a la tesis doctoral que ultima sobre la formación universitaria de los periodistas en España, la regulación profesional y los nuevos perfiles y funciones. Ha sido profesora en la Universidad Europea Miguel de Cervantes y ha ejercido el Periodismo profesional durante 15 años como redactora y colaboradora en diversos medios de comunicación españoles y extranjeros. 